\title{
Editorial
}

\section{Genetic Testing in Breast Cancer: New Standards of Care}

\author{
Christian F. Singer \\ Department of Obstetrics and Gynecology and Center for Breast Health, Comprehensive Cancer Center, \\ Medical University of Vienna, Vienna, Austria
}

Pathogenic variants in the breast cancer susceptibility genes 1 and 2 (brcal and brca2) are the strongest risk factors for the development of breast and ovarian cancer known to date. In unselected patients, they account for approximately $6 \%$ of breast and up to $20 \%$ of ovarian cancer cases. In addition, pathogenic variant genes such as palb2, tp53, pten, chek2, and atm result in a high- to moderate-risk increase in breast, and, in some cases, also ovarian, pancreatic, and prostate cancers, although mutation frequencies are significantly lower.

For many years, genetic testing in breast cancer was limited to the identification of BRCA1 and BRCA2 germline mutation carriers in families that were characterized by an apparent clustering of breast and/or ovarian cancer cases, or by a particularly young disease onset at the time of diagnosis. The finding of a pathogenic variant in one of the hereditary cancer susceptibility genes would then not only permit for a personal cancer risk estimation, but also qualify for intensified breast cancer screening, and possibly also individual risk reduction strategies. Unfortunately, despite the benefits of genetic testing, many eligible individuals never underwent testing because of limited access to genetic counseling, affordability, complicated testing criteria, and several other barriers. In particular, the selection for appropriate candidates for brca germline mutation testing is an important yet challenging clinical issue. Several risk prediction models have been developed to assist in pretest counseling by estimating the absolute risk for breast and other types of cancer, and to estimate the probability of finding germline mutations in cancer susceptibility genes. Most of these models differ in their predictive abilities, were developed in highly selected populations, and are limited by high false-negative and false-positive rates outside of high-risk popula- tions. In addition, limited family structure and lack of knowledge about family history reduce the usefulness of these models and related national guidelines.

It is somewhat surprising that our ability to identify breast cancer-predisposing germline mutations with increasingly affordable high-volume throughput tests that are now widely available has, in many ways, surpassed our ability to implement these technologies in our clinical practice in a meaningful way. This apparent shortcoming needs to be corrected in order to avoid suboptimal treatment for those needing to know their individual cancer risk and those in whom the knowledge of mutation status might have therapeutic implications.

The availability of the oral poly(ADP-ribose)-polymerase (PARP) inhibitors olaparib and talazoparib for the treatment of locally advanced and metastatic breast cancer, which are now approved as monotherapies for germline BRCA-mutated breast cancer, has turned out to be a game changer in our diagnostic algorithm: both drugs, when used as monotherapies, have demonstrated significant progression-free survival benefits compared with standard chemotherapy, and toxicities could effectively be managed by supportive treatment and dose interruptions/reductions. Furthermore, the results of the recently published OlympiA trial, in which olaparib was offered in the adjuvant setting and resulted in a significant improvement in invasive disease-free survival, will most probably result in a timely FDA and EMA approval. In addition, mutations in other breast cancer susceptibility genes that encode proteins critical for DNA homologous recombination repair have been identified as potential response predictors to PARP inhibitors in the preclinical and clinical setting. These developments will undoubtedly lead to a further increase in demand for ge- 
nomic counseling and germline panel testing as a routine procedure in this setting, and will result in an overburdening of the presently existing structures.

In order to ensure timely access to genomic counseling and mutational testing in the future, we must first increase access to genetic counseling, which is still a prerequisite for gene panel testing. Genetic counselors are an invaluable resource and can provide tremendous input; clinicians caring for breast cancer patients and their families cannot abrogate their responsibility to become educated in genetic counseling for both affected and unaffected family members. It will soon become impossible to practice medicine without genetics, and this is particularly true in the field of senology.

Secondly, to move genetic testing from a service of fered only to the few privileged who have access to highly specialized genetic centers to a service on a population level, we need to reduce pretest counseling to enable more patients to be seen by genetic counselors or educated clinicians.
Finally, we need to acknowledge that the true problem of genetic testing is not the undue stress and anxiety that is still often attributed to the knowledge of one's mutational status, but rather the fact that in Europe, hundreds of thousands of mutation carriers are still unaware of their genomic disposition and will eventually develop malignancies that might have been prevented or at least detected in a high-risk screening program. Hopefully, the prerequisite for genetic testing in an ever-increasing subgroup of breast cancer patients, who could potentially benefit from PARP inhibitor treatment, will help to facilitate low-threshold and rapid access for those in whom testing is indicated.

\section{Conflict of Interest Statement}

C.F. Singer reports expert testimony and consultancies for Novartis, Roche, Amgen, and AstraZeneca, as well as travel and grant support from Novartis, AstraZeneca, Roche, and Amgen. 